\title{
De un dispositivo cuya singularidad permite recuperar las posibilidades enunciativas en las infancias. El Consultorio Conjunto de Neurodesarrollo ${ }^{1}$
}

\section{Of a device whose singularity allows to recover the enunciative possibilities in childhood. The Joint Neurodevelopment Clinic}

\author{
Mariana Spina Rinaudo ${ }^{2}$
}

\section{Resumen}

El presente trabajo busca recuperar la experiencia llevada adelante en el marco de la Residencia de la Carrera de Especialización en Psicología Clínica, Institucional y Comunitaria durante el período 2010-2011. La práctica fue desarrollada en Consultorio Conjunto de Neurodesarrollo, dispositivo perteneciente al programa SANAR (Seguimiento Adecuado del Niño de Alto Riesgo), de la Secretaría de Salud Púbica Municipal.

Se intentará indagar acerca de los efectos y las inscripciones que, sobre la conformación subjetiva de las infancias, pueden tener los discursos que se ponen en juego (se vehiculizan) a través de los procesos atencionales propios de los sistemas de salud. Con esto se alude a los espacios de consultas e interconsultas, en los efectores públicos de primer y segundo nivel de atención, como los centros de atención primaria de la salud y los hospitales generales.

Se buscará reflexionar acerca de los mecanismos que posibilitan u obstaculizan la emergencia de la subjetividad, en poblaciones de niños y niñas con dificultades o problemáticas en el desarrollo psíquico, físico y/o cognitivo. Para situar los modos a través de los cuales algunas prácticas en salud se pueden instituir como lógicas totalizantes.

Palabras claves: Dispositivo - Procesos de atención - Problemáticas en el desarrollo Infancias - Discursos hegemónicos - Diagnósticos

\section{Summary}

The present work seeks to recover the experience carried out within the framework of the Residence of the Specialization Career in Clinical, Institutional and Community Psychology during the period 2010-2011. The practice was developed at the Joint Neurodevelopment Clinic, an institution belonging to the SANAR program (Adequate Monitoring of High-Risk Children), of the Municipal Public Health Secretariat.

An attempt will be made to inquire about the effects and inscriptions of the discours-

\footnotetext{
${ }^{1}$ El presente artículo fue reescrito sobre la base del Trabajo Integrador Final de la Carrera de Especialización en Psicología Clínica Institucional y Comunitaria (2010-2011) de mi autoría y que fuera dirigido por la Ps. Esp. Betina Chiani

${ }^{2}$ Psicóloga. Especialista en Psicología Clínica, Institucional y Comunitaria. Psicóloga en Equipos Interdisciplinarios de las Escuelas Especiales Públicas de la Provincia de Santa Fe. Región VI. marianaspina@hotmail.com
} 
es conveyed along their own attentional processes. This refers to the spaces for consultations and interconsultations, in the first and second level of the public effectors, such as primary health care centers and general hospitals.

It will seek to reflect on the mechanisms that enable or hinder the emergence of subjectivity, in populations of boys and girls with difficulties or problems in psychic, physical and/or cognitive development, to locate the ways through which some health practices can be instituted as totalizing logics.

Keywords: Device - Process of attention - Problems in the developmentchildhood Discourses hegemonic - Diagnoses

\section{Introducción}

El devenir en la constitución psíquica de los niños y niñas supone procesos y construcciones que implican la relación con los otros, las instituciones y los discursos que se ponen en juego en dichos espacios.

El recorrido que se intenta diseñar con el presente trabajo surge a partir de problematizar la articulación entre la clínica y la institución, en el abordaje de niños y niñas con problemáticas en el desarrollo.

El Consultorio Conjunto en Neurodesarrollo puede pensarse al modo de un dispositivo artesanal, como lo propone Gaston de Souza Campos (2001), que permite ubicar y problematizar el recorrido que realizan niños y niñas desde los procesos atencionales $\mathrm{y}$, de esta manera, poder interpelar las marcas que producen en la construcción subjetiva aquellos discursos que aparecen instituidos a partir de estos lugares.

Juan Vasen (2011) sostiene que:

ningún enfoque del sufrimiento infantil debería soslayar que la subjetividad se entrama centralmente, a partir de las relaciones fantasmáticas y deseantes con los otros y con las estructuras anónimas que el sistema social produce y reproduce (p. 37)

Que una consulta se disponga de manera conjunta, con la presencia de un psicoanalista posibilita un abordaje interdisciplinario, una de cuyas direccionalidades es trabajar desde un abordaje preventivo en el sentido de poder interrogase acerca de lo que le pasa a un sujeto infantil que consulta, suspendiendo juicios anticipatorios.

Vasen considera que se trataría de abandonar posiciones que tiendan a clasificar, a configurar serializaciones, siendo que para el psicoanálisis, siempre hay algo de lo humano que se escapa, que es inclasificable. "La diferencia entre clasificar y diagnosticar con respecto al saber es que la clasificación se detiene en la particularidad. El logro clasificatorio es la inclusión en una serie" (Vasen, 2011, p. 23)

En estos desarrollos el concepto de prevención está pensado y entendido como el abordaje temprano, oportuno e integral; en cuanto a poder despegar al sujeto de su falla real, de la patología, para dar lugar a una relación con la falta singular.

Apelar a la participación y al derecho de los sujetos en incluirse e involucrarse en los procesos de atención en salud, es dar lugar a la enunciación, al contexto en el cual emergen las problemáticas, los conflictos. Es pensarlo en un entramado histórico singular, social, cultural y político.

Un interrogante que es interesante recuperar, a la luz de estos desarrollos, remite a pensar en cuántas ocasiones el encuentro con el infantil sujeto, que padece o sufre alguna afección en su desarrollo, confronta a los profesionales con elementos desconocidos, remitiendo a tiempos propios de mayor inermidad, generando muchas veces lugares de expulsión, de rechazo, de negación.

De este modo, aquí se intentará visibilizar, 
a través de las delimitaciones conceptuales y el recorrido por algunos fragmentos clínicos, ciertas modalidades que adquieren las prácticas en salud y los procesos atencionales, desde la singularidad que produce el encuentro con el infantil sujeto, dificultad que se reduplica si este niño/a presenta alguna patología orgánica o se encuentra en situación de discapacidad.

La premisa a desplegar sería que posicionamientos cerrados conllevan un debilitamiento subjetivo en el sujeto que consulta.

\section{Historización acerca del dispositivo}

El Consultorio Conjunto de Neurodesarrollo se incluye desde sus orígenes en el Programa SANAR (Seguimiento Adecuado del Niño de alto Riesgo), de la Secretaría de Salud Pública Municipal de Rosario, elaborado en el año 1992. Este programa propone realizar el seguimiento y control de niños considerados de "alto riesgo", que egresan de los Servicios de Neonatología de las maternidades municipales, hasta los ocho años, con el fin de detectar precozmente posibles patologías y/o alteraciones en el desarrollo y ofrecer su correspondiente tratamiento.

El SANAR sostiene que la detección pre$\mathrm{coz}$ de las patologías y el abordaje temprano en niños y niñas con problemáticas en su desarrollo propician la prevención de secuelas discapacitantes y, en el caso de que presenten alguna lesión orgánica, o retraso en las pautas madurativas, reduce el impacto para mejorar sus condiciones y calidad de vida. Como espacio de consulta, el Consultorio de Neurodesarrollo comparte con otras disciplinas, como neurología, pediatría, trabajo social (incluidas en el Programa de Seguimiento) la tarea de realizar el seguimiento de los niños y niñas considerados de "alto riesgo".

Desde el programa se considera niños/as de riesgo, a aquellos/as que habiendo estado internados/as en su período neonatal en
Unidades de Cuidados Intensivos Neonatales, tienen un riesgo elevado de secuelas, como aquellos con antecedentes de prematurez con bajo peso al nacer, depresión grave al nacer, asistencia respiratoria mecánica, sepsis/meningitis, incompatibilidad sanguínea $(\mathrm{RH}, \mathrm{ABO} \mathrm{u}$ otras que hayan requerido exanguinotransfusiones $\mathrm{y} / \mathrm{o}$ transfusiones intrauterinas), poliglobulia sintomática $\mathrm{y} / \mathrm{o}$ tratamiento con sangriatransfusión, patología neurológica (convulsiones, hemorragias intracraneanas), trastornos metabólicos graves, infecciones intrauterinas, patologías quirúrgicas complejas, malformaciones o síndromes genéticos severos y condiciones de vulnerabilidad social.

En el año 2001, luego de realizar algunos análisis acerca de la adhesión al programa y relevar una alta deserción por parte de los pacientes, se reordenan ciertos modos de funcionamiento y el programa comienza a tener otras implicancias: los equipos referenciales de los territorios se ocuparían del seguimiento periódico del niño/a, y el SANAR funcionaría como espacio de interconsulta en los hospitales.

\section{¿Cuál es la potencialidad de dicho dis- positivo?}

El Consultorio Conjunto de Neurodesarrollo se ha ido diseñando a lo largo de los años de un modo artesanal, adquiriendo rasgos propios en el abordaje de las situaciones y problemáticas e instituyendo modalidades de atención a partir de las articulaciones que se han ido generando con actores y profesionales de diversos efectores de salud. Los lazos transferenciales construidos en este tiempo de trabajo con los equipos de salud territoriales, constituyeron una referencia para la interconsulta con el dispositivo, no sólo de aquellos niños/as que han atravesado una historia neonatal muy comprometida sino también de toda otra población infantil que ya sea por 
manifestar dificultades o padecer algún sufrimiento pueda estar interrogando a los actores de los servicios de salud.

El malestar que niños o niñas puedan estar desplegando supone un interrogante, una pregunta, es en estas situaciones donde se van configurando escenarios posibles para la interconsulta.

No se puede dejar de señalar que el Consultorio Conjunto de Neurodesarrollo es un dispositivo que forma parte de la red de atención pública de Salud Municipal, y como tal está concernido respecto a las coordenadas que afectan los procesos atencionales en salud.

Desde nuestro entender las prácticas que sostienen los efectores públicos de primer y segundo nivel de atención pertenecientes al sistema de Salud Pública, constituyen un entramado discursivo que, en ocasiones, puede poner en cuestión la emergencia de un sujeto. Esto puede significar un atolladero aún mayor en el caso singular de niños y niñas que presentan dificultades en su desarrollo o atraviesan por alguna situación de discapacidad.

Souza Campos (2001) entiende que:

la estructura tradicional de los servicios de salud refuerza el aislamiento profesional y reproduce la fragmentación de los procesos de trabajo, manteniendo sin cuestionar la hegemonía del poder médico.

En general cuando interviene un especialista o profesional en un caso, lo hace de manera vertical, con acciones separadas unas de otras. Es una sumatoria de procedimientos realizados "al lado" uno de otros, con la suposición que al cumplir cada uno su parte estaría garantizada la cualidad del todo. Casi no hay integración horizontal permanente entre los distintos saberes y prácticas, y cuando las hay, le correspondería al médico compatibilizar las diferentes lógicas involucradas en el proceso de trabajo. (p. 30)

Resulta pertinente destacar la existencia de determinadas situaciones cuando, en las consultas, la especialidad o superespecialidad se expone como un único saber ante el sufrimiento de un sujeto y coagula todo otro sentido, anulando las posibilidades enunciativas de los sujetos que consultan. De este modo, se ponen en escena modelos clásicos de salud. El seguimiento sostenido desde estas lógicas genera modos alienantes y desimplicantes en las poblaciones respecto de su padecimiento.

Campos propone pensar los dispositivos ${ }^{3}$ como facilitadores de un nuevo orden organizacional que estimulase el compromiso de los equipos con la producción de salud.

Advertidos de que el Consultorio Conjunto de Neurodesarrollo, en tanto dispositivo inserto en las redes de poder y de sentido, la inquietud gira en torno de los modos de producir una discontinuidad en este entramado discursivo.

\section{¿Cuáles son las coordenadas que su- pone?}

El Consultorio Conjunto está compuesto por instancias que podemos mencionar como la consulta propiamente dicha, y los momentos de re-trabajos. Está constituido por una médica pediatra neonatóloga (especialista en neurodesarrollo y responsable del consultorio), y una psicóloga que acompaña el dispositivo; al tiempo que se va conformando con la presencia de psicólogos que participan del espacio en el marco de la residencia desde la Carrera de Especialización en Psicología Clínica Institucional y Comunitaria y residentes de la $\mathrm{Ca}$ -

\footnotetext{
${ }^{3}$ Campos (2001) retoma a Baremblit en cuanto a que "dispositivo es un montaje o artificio productor de innovaciones que genera acontecimientos, actualiza potencialidades e inventa lo nuevo radical” (p. 144).
} 
rrera de Especialización en Psicología General y Familiar que realizan sus rotaciones por el espacio.

En las instancias de re-trabajo participan psicólogas, tutoras de la residencia de la Carrera de Especialización antes citada que supervisan las situaciones.

Se propone como un dispositivo de evaluación conjunta, en cuanto intenta poner en acto un proceso diagnóstico desde la complejización de lecturas dada por la conformación de diversas praxis y campos teóricos.

Al mismo tiempo, también es un espacio de formación para los residentes de las carreras anteriormente mencionadas, dentro de la lógica de la atención primaria de la salud.

El Consultorio Conjunto intenta conformar una referencia para los equipos de salud territoriales, quienes apelan desde la interconsulta al trabajo con el dispositivo. Así mismo, es un espacio de interconsulta para las especialidades que funcionan en niveles de mayor complejidad, como los hospitales generales.

El dispositivo puede operar, en ocasiones, como apoyo matricial especializado; en otras, como un espacio para reordenar los procesos de atención, y de esta manera intentar evitar los puntos de excesiva instrumentación de los sujetos que consultan (los niños y las niñas que llegan a las consultas).

El objetivo que se proponen las prácticas en el marco del dispositivo, es ofrecer instancias para articular las derivaciones, en algunos casos y, en otros para generar cierta estabilidad y luego derivar, situando la prevención como lineamiento, como contrapartida de toda búsqueda de normativización y clasificación de la población.

Castel (1984) advierte acerca de una orientación que desarrollan ciertos dispositivos médico-psicológicos que, al permitir una disociación radical entre el diagnóstico y la asistencia, decanta la práctica en puro peritaje, convirtiéndose de este modo en instrumentos de gestión diferencial de las poblaciones.

\section{Las vicisitudes por las que atraviesan los niños y niñas con dificultades en el desarrollo}

Resulta necesario poner en tensión el concepto de vicisitudes, acudiendo a la pregunta por las condiciones de posibilidad para que algo del orden de un acontecer tenga lugar en la estructuración subjetiva de un niño. Es decir, para que un niño o niña con problemáticas en su desarrollo o con una historia neonatal compleja, no quede sujeto a dichas marcas, y tenga la oportunidad de transitar como una aventura su constitución subjetiva, pensando esto no como algo dado, sino como algo a conseguir.

Señala Jerusalinsky (2005):

Recordemos que lo madurativo, le hace límite al sujeto pero no lo determina ni lo causa. El surgimiento del sujeto del deseo, hablante, sexual, sujeto del inconsciente, sujeto histórico, depende de la simbolización del cuerpo en los primeros tiempos de vida y de cómo se sortean los límites a dicho trabajo de simbolización, por el otro y por el niño, ya sean límites biológicos que impermeabilicen la entrada del lenguaje o límites del significante en hacer su trabajo, límites en ese otro primordial para responder al desamparo del cachorrito humano recién llegado a este mundo que se reduplican en un niño con problemas orgánicos.

De este modo en el trabajo con niños que sufren alguna patología o presentan una dificultad en el desarrollo, nos enfrentamos con la pregunta por las condiciones de posibilidad para la entrada del significante y su función de forma encarnada, no anónima para el nacimiento del sujeto de deseo y los objetos en el deseo. (p. 33) 
El Consultorio de Neurodesarrollo, como parte del Programa de Seguimiento desde los objetivos que le dan origen y consistencia, intenta articular operatorias para un abordaje y una mirada preventiva, generando condiciones para el acontecimiento.

Dice Bruner (2008)

El desarrollo es la carretera que se va construyendo, organizada desde el material significante, donde tienen lugar y transcurren para el sujeto los trayectos, viajes y virajes del deseo y sus instrumentos, desde el nacimiento hasta la muerte. Este desarrollo-carretera funciona, al mismo tiempo, como límite último de dicho transcurrir. (p. 39)

\section{Acerca de la inclusión del psicoanalis- ta en el Consultorio Conjunto de Neu- rodesarrollo. La interconsulta}

La presencia del psicoanalista, en el Consultorio Conjunto abre un sendero, en tanto que como señala Ginette Raimbault (1985) el sufrimiento del paciente y sus síntomas escapan a la posibilidad de conocimiento biológico del cuerpo. Los síntomas de un paciente están en relación con el hecho de que cada individuo habla en el interior de unas coordenadas, de unas significaciones, de unas posiciones bien precisas que distinguen a cada cual de todos los demás y que hacen que los síntomas sean inaccesibles a unas generalizaciones.

Como entiende esta autora el cuerpo no sólo se encuentra regulado por hechos anatómicos, fisiológicos o biológicos, sino que un cuerpo se encuentra atravesado por las dimensiones del sufrimiento y del goce.

La inclusión del psicoanalista en el dispositivo favorece la dimensión del discurso en las prácticas de atención, introduciendo de este modo la diferencia entre el cuerpo en general y tal o cual cuerpo en particular.
La participación del psicoanalista en el Consultorio Conjunto introduce la posibilidad de una intersección en relación con lo discursivo, posibilitando poner en interlocución determinados enunciados, que permitan reabrir aquellos procesos que en ocasiones corren el riesgo de quedar suturados. En ese marco, ¿cuáles son las posibilidades del psicoanalista en una consulta conjunta, que en primera instancia sostiene las coordenadas médicas, de introducir la dimensión del discurso, contemplando el planteo de Raimbault (1985) respecto de que el cuerpo se encuentra cifrado y marcado por el lenguaje, por el orden imaginario y simbólico? Tal como ella misma señala, el poder de la palabra tiene efectos sobre el cuerpo.

¿Cómo situar algo del orden de "un estar psicoanalista" (Ulloa, 1995), suspendiendo juicios a priori, en los casos que llegan al consultorio? Esta posibilidad interpela de modo significativo en aquellos casos de niños y niñas que por su historia singular requieren de la intervención de múltiples disciplinas como neurología, traumatología, genética, fisiatría, nutrición, neurocirujía, oftalmología, entre otras.

Retomando algunos planteos de Silvia Grande (2000) podemos señalar que el modo en que se piense la interconsulta refleja y/o da cuenta del modo en que se produce la institución y la clínica

Silvina Gamsie (1998) señala que el terreno de la interconsulta sigue constituyendo el campo más propicio para la interlocución entre la medicina y el psicoanálisis, ya que, según esta autora, el mismo llamado supone un sujeto: el médico que se ha interrogado por ese paciente que no responde de la manera esperada. De lo contrario, no nos llamaría.

Gamsie explica que la finalidad, lejos de agotarse en la realización de un diagnóstico diferencial, se centra en la posibilidad de operar sobre la demanda de los equipos médicos, abriendo paso a la interrogación de su males- 
tar. Lo que supone una ardua tarea, en épocas en las que los pedidos suelen ser formulados de manera tal, de esquivar cualquier posibilidad de implicación por parte de quien los formula

La modalidad que el dispositivo de Consultorio Conjunto adquiere en cada situación toma forma a partir de la lectura clínica de las demandas y de cómo se ubican los profesionales en relación a los pedidos y el tipo de respuestas que se ofrecen.

De esta manera, en algunos casos, se articulan vías para las derivaciones; en otros, se crean los escenarios para generar cierta estabilidad en las situaciones que llegan a la consulta y luego derivar, en todos los casos, indagando acerca de las condiciones de posibilidad para la construcción de una interconsulta.

En tanto el dispositivo se ordena en relación con las lógicas de atención primaria de la salud en redes, promueve la participación y la inclusión de los usuarios en los procesos de atención, intentando garantizar en cierta medida estrategias de promoción y prevención en salud.

\section{¿Cómo conformar y hacer viable un diagnóstico que permita al sujeto de- cir sobre lo que le acontece?}

La pregunta que da título a este apartado muestra la direccionalidad de las prácticas sostenidas desde el dispositivo en tanto aparece con insistencia en aquellas ocasiones en la cuales el pedido de interconsulta connota un saber cerrado, más cercano al encargo social.

Cuando las consultas reflejan un pedido para la formulación de un diagnóstico que describa y enmarque lo que a un niño o niña le pueda estar aquejando y establezca intervenciones a seguir, reparamos que nos encontramos ante posicionamientos en los que se deslizan los discursos médicos clásicos. Estos escenarios requieren de una praxis que permi- ta sostener las problematizaciones y la complejización.

Es interesante recuperar las elaboraciones y desarrollos propuestos por Silvia Grande ${ }^{4}$ para situar que prácticas médicas de este orden se instauran como instituciones totalizantes, cuando se constituyen en un único sentido, coagulando el advenimiento de otros sentidos.

Grande (2010-2011) nos propone pensar que el discurso médico hegemónico hace institución allí donde pretende sostener un único saber y obtura las posibilidades de enunciación de los sujetos.

Cuando las demandas que llegan al Consultorio Conjunto provienen, como plantea Liliana Baños (2012, p 62) "no de lo que no funciona sino de lo que se supone que debe funcionar", en este escenario nos encontramos con el obstáculo, al modo de un "encargo social".

El encargo social toma la figura de un diagnóstico a la manera de una etiqueta que, lejos de trazar vías para posibles abordajes, obtura todo recorrido posible.

¿Qué haría el enfermo con un diagnóstico? El diagnóstico está destinado a otros. El hecho de formular un diagnóstico psiquiátrico desaloja entonces al enfermo de su posición de sujeto, lo somete a un sistema de leyes y de reglas que escapan a su comprensión e inaugura así un proceso que desembocará lógicamente en medidas de segregación" (Mannoni, 1976, p. 22).

Es pertinente considerar estas prácticas situando el obstáculo como estructural, tal como lo propone Liliana Baños (2012), aquello que facilita o impide, que permite o

\footnotetext{
${ }^{4}$ Clases teóricas dictadas durante lo seminarios de Abordajes Institucionales I y II en la Carrera de Especialización en Psicología Clínica, Institucional y Comunitaria cohorte 2010-2011
} 
dificulta, el punto de articulación y de apertura hacia el trabajo con los otros.

Los momentos de retrabajo, en los cuales psicólogas tutoras supervisan y acompañan las prácticas, constituyen una instancia significativamente valiosa en el escenario del Consultorio Conjunto. Introducen tiempos para una reflexión compartida, para revisar las intervenciones, para reabrir a través de preguntas aquellas situaciones, cuando un sentido aparece coagulado, permitiendo conmover ciertos saberes y destotalizar lo que aparece instituido.

Otra de las instancias que se promueve desde el dispositivo es el encuentro con los profesionales de los equipos de salud, de los efectores territoriales y, así mismo, con otros actores que sostengan las intervenciones en el trabajo con las poblaciones infantiles que llegan a las consultas. Estos escenarios posibilitan la reflexión, la elaboración conjunta, la complejización de las situaciones, permitiendo una construcción colectiva en la producción de salud de los sujetos.

El oficio, la tarea sostenida con otros, habilita operatorias que permiten interpelar las instancias constatativas, cuando lo constatativo impide el paso, la articulación del sufrimiento en una queja, que favorecería una mayor movilidad y la emergencia del sujeto (Baños 2012).

Es oportuno señalar que lo que constituye el obstáculo, la dificultad en la práctica analítica, no sería la institución, sino lo institucionalizado, que en ocasiones puede instalarse en distintos discursos, como por ejemplo "estas madres no entienden", "a estos chicos les cuesta aprender".

Señala Baños (2012) que el psicoanálisis permite reabrir estos interrogantes ubicando el punto donde cada uno de estos discursos ha hecho institución, porque la institución cierra, responde renegatoriamente a la pregunta que la funda.

La posibilidad de un niño y de su madre, de constituirse en sujetos de la enunciación, pasa por habitar el lenguaje, ser soporte de la palabra (Mannoni 1972).

Recuperando algunas conceptualizaciones de Grande (2010-2011) se puede situar querescatar algo del orden del saber es un intento de dar sentido. La autora considera que el saber es un modo de lazo al otro. Comprender, saber, construir escenas para que algo de lo encubridor funcione. Saber es un recorte.

Apelando a las consideraciones de Vasen (2011), se trataría de mantener la enunciación como historicidad y contradicción en lugar de convertir la nominación en nomenclatura. El lenguaje, en tanto acto de enunciación, para él, es lugar de la verdad. Las verdades hacen acto de presencia en un espacio de prácticas donde ese sufrimiento se despliega. En contraposición, aparecen las clausuras taxonómicas.

Si retomamos la pregunta por el o los diagnósticos, sostenemos que lo esperable es que se pueda dar lugar a la historia de ese niño o niña, en una trama afectiva y social, y situada en un contexto, para pensar las inscripciones que los habitan.

\section{Comenzar a contar- $\mathrm{se}^{5}$}

A continuación se describirán diferentes momentos e instancias de trabajo, en cuanto a las estrategias articuladas en una situación de una niña de siete años. En el caso de esta niña, podemos referir un compromiso orgánico muy tempranamente en su vida, ya que su historia neonatal ha sido muy complicada. Se pueden mencionar algunas referencias: nacimiento prematuro extremo con bajo peso al nacer, depresión grave, diversas complicaciones en lo orgánico. Internada en sala de Neo-

\footnotetext{
${ }^{5}$ Se han tomado los resguardos éticos correspondientes a los fines de proteger la identidad de los concernidos.
} 
natología durante casi tres meses, requirió de múltiples intervenciones y de cuidados intensivos en el tiempo de internación hospitalaria.

$\mathrm{Al}$ alta continúa los controles periódicos (cada dos o tres meses) con el espacio de Neurodesarrollo. En la historia clínica pueden leerse los elementos de la reseña, que reflejan un desarrollo esperable y dan cuenta de una buena evolución en lo cognitivo, adquisición en las pautas madurativas acordes a los tiempos cronológicos: la marcha, la motricidad, el lenguaje, la aparición del juego. La médica señala en la historia clínica que "el compromiso orgánico de su historia neonatal se encuentra superado".

En el caso de Micaela se enuncia un acontecimiento en el Consultorio Conjunto, que no había sido dicho en ninguna de las consultas, ni con la médica de referencia del centro de salud, ni en el espacio con el psicólogo, aunque era un dato que estaba escrito en la historia clínica familiar.

\section{Consulta con Neurodesarrollo}

Es en esta instancia en la cual me incluyo por primera vez. La niña viene asistiendo con periodicidad a controles con Neurodesarrollo en el marco de seguimiento desde el programa SANAR. En esta oportunidad llegan a la consulta la madre y la niña, con la referencia de la médica del Centro de Salud.

Referencia: "Niña de 7 años, 10 meses con antecedentes de prematurez extrema y múltiples complicaciones internada en neonatología, realiza los controles de salud regularmente, y se encuentra en tratamiento con psicología por trastornos de conducta.

La niña presenta problemas de aprendizaje y en la comunicación con sus pares, con una importante inhibición hacia lo desconocido. En el aula y en la consulta no habla ni contesta consignas. No cumplió con todos objetivos de la currícula escolar.
Dra nos interesaría su evaluación, dado que usted realiza seguimiento de la niña y ver sus avances y si cree necesario realizar algún tipo de estudio para descartar patología orgánica. A su disposición”. La referencia está firmada por su médica de centro de salud.

La mamá relata que el embarazo fue gemelar y que una de ellas falleció a los pocos días de nacida. Refiere que esto no lo cuenta mucho: "Entre la familia hablamos todo, pero no contamos al resto”. Agrega: "Los papás tenemos un hijo cada uno de otro matrimonio, más grandes y la gente pregunta si son mellizos".

La niña permanece en una actitud tranquila, apocada, realiza las consignas y actividades lúdico cognitivas que la doctora va proponiendo, en un tono de voz bajo, casi susurrando, pero atenta a lo que su mamá conversa.

La mamá continúa refiriendo que la consulta es porque ella no habla con el resto, $y$ dice: "En la escuela hace las cosas pero no habla, ahora menos." Y se pregunta "¿La culpa la tendré yo por sobreprotegerla?" También si la sobreprotege porque falleció la hermana. Esto es lo que no se cuenta.

"Yo no veo el problema, para mi no tiene nada, los demás me dicen”, agrega.

"Micaela pregunta por su hermana. Siempre se acuerda en los cumpleaños, pregunta si su hermana lo festejará en el cielo". En este punto la mamá comienza a llorar.

La niña está atenta, en este momento mira hacia donde hay unos peluches en la camilla y se acerca a ellos. Comienza un juego, toma el payaso y lo coloca frente a otros muñecos. Lo hace saltar, bailar, hacer piruetas. El resto de los muñecos lo miran, permanecen en una situación pasiva, meros espectadores, de las "payasadas".

En esta oportunidad nos comunicamos con el psicólogo que atiende a la niña para comentar brevemente la consulta, y comenzar un intercambio. Él refiere que asiste desde el año pasado, pero con muchas irregularidades 
y señala: "No están de acuerdo", en referencia a que la madre no acuerda con que su hija realice tratamiento psicológico.

En esta primera consulta se confecciona una contrarreferencia escrita dirigida al equipo del centro de salud y se la vuelve a citar para otra consulta.

\section{Siguiente consulta con Neurodesarrollo}

La mamá refiere que Micaela comenzó bien la escuela (es fecha de inicio del año escolar). "Con la maestra nueva se está conociendo más, la saluda, le da un beso. Me sorprende. Tiene dos docentes, con la que no habla es con la docente del año pasado. Ella es quien me comunica de los problemas en la cabeza que tenía mi hija. Esto me molestó", dice y llora al recordar el episodio. y continúa: "Yo se que la quiere, estamos todo el tiempo hablando de la maestra. Es el tema de conversación. Estamos torturándola, ahora estoy entregada.Hice todo lo que está a mi alcance, ¡es demasiado! Traerla a todos los médicos, no está enferma”.

Comenta que se reunieron el equipo de Salud y las docentes, y le dijeron que querían reunirse con "la doctora", (en referencia a la neurodesarrollista) para ver cómo trabajar.

"Yo vi cambios, me angustia. Yo perdí una hija, y no quería que ella tenga problemas. No tiene problemas", afirma.

Refiere también que la docente le dijo que si era una nena normal, tenía que repetir de grado pero si tenía dificultades, pasaba con adecuaciones. Asimismo, comenta que la pediatra ha hablado con el psicólogo acerca de unos cambios en los dibujos que ocurrieron posteriormente a la consulta con neurodesarrollo.

Mientras tanto, la niña ha estado jugando con diferentes objetos, ha hablado más, arma una especie de pista por la que circulan autos, luego, un auto-tren que se va de viaje, lo carga con cosas, ropa, comida. "Se preparan para un viaje", dice. Saludan a una muñeca que es la que se queda.

En esta consulta y situando la pregunta de la mamá respecto de los problemas que aquejan a la niña, la médica realiza una devolución donde despeja que no se trata de problemas neurológicos, que su historia neonatal se encuentra superada, pero que hay que atender las dificultades ya que lo que implica una inhibición en la oralidad, sea de orden emocional o subjetivo, puede generarle otras dificultades en el aprendizaje.

En las instancias de re-trabajo posteriores, se ubican puntualizaciones referidas a la situación, en cuanto a tratar de no poner sentido a lo que le pasa, sino abrir en lugar de cerrar, y trabajar en el marco de una devolución al equipo de referencia en relación a que pareciera que algo queda expulsado, que habría algo más. Se conviene realizar una reunión con el equipo que atiende a la niña, en el territorio.

En la reunión con el equipo de centro de salud en la que estaban presentes la médica generalista y el psicólogo que lleva adelante su tratamiento, se efectúan algunos intercambios en cuanto a reconstruir la historia neonatal de la niña, el seguimiento realizado, y las últimas evaluaciones con neurodesarrollo. Por otro lado, el equipo del centro de salud refiere algunos indicadores y dificultades en el trabajo con la paciente y su familia.

Se acuerda realizar una reunión conjunta con el equipo educativo de la niña y se intenta despejar con la médica que no se trataría de una afección neurológica, ya que la evaluaciones clínicas están acordes con su tiempo madurativo, sino que habría otros aspectos que se juegan en la problemática: la historia de la niña, los intercambios primarios, la fantasmática materna. En cuanto a la inhibición en el habla, se ubica como hipótesis que la niña va a oponerse a todo aquel que intente dirigirse a ella. Por su parte, el psicólogo que lleva adelante el tratamiento de la niña señala que tiene 
algunas dificultades en cuanto a la asistencia y al trabajo con la familia, ya que se encuentran muy negados.

En la reunión acordada con la escuela de la niña participan el equipo del centro de salud, el equipo del dispositivo de Consultorio Conjunto de Neurodesarrollo, docente y directivos de la escuela. Allí se exponen algunas cuestiones advertidas en la consulta para intentar dar lugar a cierta historicidad, y reubicar la problemática de la niña en otro orden. Se acuerda que el equipo educativo pueda recurrir al equipo del centro de salud ante algunas inquietudes, y que se continuará desde el Consultorio de Neurodesarrollo con las consultas que sean necesarias.

¿Qué podemos reconstruir en este fragmento clínico?

En primer lugar, ubicar algunos interrogantes que nos permitan recortar la situación: ¿Qué recorridos atraviesa esta niña desde su historia neonatal, superada desde el aspecto orgánico y funcional? ¿Cómo se entrecruzan las singularidades y condiciones de nacimiento con las circunstancias de su desarrollo afectivo y subjetivo en la trama familiar?

En tanto es posible constatar que el seguimiento realizado con la niña durante sus primeros años no señalaba complicaciones significativas, ¿cuáles son los aspectos que se jugaron en el intercambio primario con su mamá, en relación con la historia de la hermana fallecida?

¿Qué es aquello que no puede contarse a otro? El fallecimiento de la hermana nunca había sido dicho, o escuchado, de tal manera que posibilitara una pregunta, que permitiera enlazar con aquello que aquejaba a la niña, y articular en una trama discursiva, en tanto aparecía solo como un dato.

¿Qué es aquello que la mamá no puede contar? ¿No puede contar a su hija fallecida como una hija más? ¿Cuáles son las implicancias de esta historia en aquello que Micaela conforma como una posible sintomatología leída en el plano escolar y del cual la médica se hace eco? La sintomatología retorna al modo de un silencio, de algo mudo, acallado, algo no puede contarse, y por tanto descontarse, en el plano fantasmático materno.

Desde el dispositivo de neurodesarrollo se ofertaron diferentes instancias para intentar construir una problemática que permitiera realizar intervenciones oportunas y de modo integral ¿Por qué la reunión con el equipo de salud? ¿Por qué participar en la reunión con las docentes?

Se fueron acompañando y sosteniendo las evaluaciones para ir conformando de manera conjunta otra mirada, que permitiera dar lugar a lo posible en los recursos subjetivos y cognitivos de la niña.

Es posible situar que ciertas intervenciones promovidas desde el dispositivo de Neurodesarrollo permitieron movimientos para comenzar a trabajar de otra manera con la niña e interpelar la necesidad de un diagnóstico cerrado, esto que intenta impulsar la institución educativa, y de la cual se hace eco el equipo del centro de salud, situando las manifestaciones de la niña en otro orden diferente al orgánico, para enlazarlas con su historia singular.

\section{Recorte de algunos casos derivados por un mismo efector de salud y las instancias que fueron propuestas para el abordaje de los mismos ${ }^{6}$}

En este apartado el interés es recuperar las instancias y las estrategias construidas en el abordaje de algunas situaciones, para visibilizar las modalidades que fue asumiendo el dispositivo en el trabajo con los otros.

En los momentos de re-trabajo nos dis-

\footnotetext{
${ }^{6}$ Se han tomado los resguardos éticos correspondientes a los fines de preservar la identidad de los concernidos
} 
pusimos a reconstruir las lecturas acerca de las referencias escritas enviadas por los médicos que recurrían al dispositivo. Algunas consideraciones sobre los informes aludían al modo en que estaban narrados esos escritos, en los cuales se recortaban aspectos aislados o fragmentados de las situaciones. Pesquisamos que había una inquietud en que se evaluara y constatara, desde el dispositivo, el grado de afectación orgánica y/o cognitiva que determinara la necesidad de gestionar o tramitar certificados, becas, pases.

Frente a estas solicitudes, surge la inquietud por proponer un escenario de trabajo conjunto con los profesionales del equipo, que permitiera devolver al modo de preguntas, elementos en las historias de los niños y niñas que intentaban quedar por fuera de la problematización, apelando a la reflexión conjunta.

Ofrecer oportunidades de encuentro con los profesionales del equipo, previos a la llegada a la consulta del niño o niña que iban a derivar, posibilitó introducir otros tiempos, comenzar a conformar conjuntamente las problemáticas y diseñar las estrategias de manera articulada.

En este escenario, recurrir al trabajo con los otros, a partir de convocarnos en encuentros con los profesionales de los equipo de salud que sostenían el seguimiento y los tratamientos, posibilitó introducir elementos propios de la interconsulta, entendida como campo de entrecruzamiento de discursos y problemáticas.

\section{Escenas de trabajo con Axel}

En el marco de un encuentro entre las participantes del dispositivo de Neurodesarrollo y profesionales del Centro de Salud se presenta la situación previamente a la llegada del paciente a la consulta. La médica y la psicóloga que comenzaba a trabajar recientemente con el niño refieren algunos aspectos: la médica ha notado una facie particular con algún fenotipo y se interroga por este rasgo en el sentido de que pudiera haber afectación cognitiva. Señalan que se trata de una derivación realizada por la escuela, que pone a la familia la condición de un tratamiento. La escuela indica en una nota enviada a su médica de referencia: "presenta apatía, le cuesta retener conceptos".

Las profesionales refieren que la familia asiste de manera irregular a las consultas con salud, excepto cuando la escuela lo pide como condición. La mamá solicita turnos y luego, no concurre.

Por otra parte comentan que la mamá ocultaría quien es el padre real del niño, ya que de joven tuvo otra pareja. Actualmente convive con una pareja con la cual tuvo dos hijos más. Se queja de que el papá de los chicos hace diferencia entre ellos ya que se lleva a los otros dos hijos y no a Axel.

Las profesionales señalan que madre e hijo están pegados, que el niño es feliz cuando no concurre a la escuela, porque se queda con la mamá.

En la escuela pega, aunque dice no querer hacerlo, pero que no podría hacer otra cosa. Pega porque insultan a su mamá.

Luego refieren que la abuela del niño habría dicho en el centro de salud, de modo impulsivo, que la madre de Axel sería producto de una violación. Además, comentan que la mamá del niño se corta, y todo la desborda.

La pregunta del equipo se orienta hacia las posibilidades madurativas y cognitivas del niño, para despejar posibles síndromes o disfunciones.

\section{Consulta con Neurodesarrollo}

El niño tiene 9 años y asiste a la consulta con su mamá. Realiza las actividades y consignas que la médica le va sugiriendo.

Al comenzar a hablar, la mamá puede ubicar ciertas referencias en cuanto a lo que le 
pasa a su hijo: "El problema es la conducta, empezó cuando él quería saber por su apellido, sus hermanos tienen otro apellido" (El niño lleva el apellido materno). "Está todo mezclado. Yo también a los 12 años me preguntaba por mi apellido. Mis hermanos tenían otro. Supe, después, que fui criada por mi abuelo y su pareja. Mi mamá era mi hermana, me traía regalos, yo no lo podía creer. Después tuve una buena relación".

En la evaluación la médica pudo establecer que en el área de las matemáticas el niño se encuentra bien, acorde a su edad. La dificultad aparece en lengua, donde escribe todo pegado. Sugiere realizar actividades, fútbol, relacionarse con amigos.

El niño se muestra interesado por unas cartas de DragonBall.

En esta consulta fue posible pesquisar que el niño se encuentra atravesado por una problemática familiar que lo aqueja, y esto genera obstáculos a la hora de realizar producciones propias. Si bien se encuentran afectadas algunas funciones cognitivas, esto podría estar anudado a determinados malestares y sufrimientos psíquicos. En la consulta no se puede establecer una discapacidad, sino cierta dificultad adquirida que se entrama desde su historia y sus vivencias.

En esta oportunidad, el dispositivo permitió orientar la escucha, como señala Ginette Raimbault (1985), para articular una escucha descentrada en relación con la demanda.

\section{Escenas de trabajo con Mateo}

En esta situación, la inquietud formulada por el equipo era despejar cierto grado de afectación cognitiva, y señalar las causas posibles de las problemáticas del niño.

Mateo tiene 12 años y asiste a la consulta acompañado por su mamá, derivado por su médica de referencia. También realiza tratamiento psicológico.
Mientras el niño resuelve las actividades que propone la médica, algunas con dificultades, la mamá realiza algunos relatos: "mi primogénita nace muerta". "Yo no quise esperar para tener otro bebé, y mi marido sí, entonces nos separamos. Yo quedo embarazada enseguida de otro hombre".

Respecto del niño, comenta: "En casa hace todo: lava, hace la comida cambia a la hermana más pequeña. En la escuela no aprende. Fue a la fonoaudióloga, pero yo le dije que no adelantaba. Fuimos con el neurólogo quien nos sugiere la necesidad de una medicación, yo veía que no avanzaba".

Mateo tiene dos hermanos, uno de 10 años y una hermana de cinco.

El niño habla muy rápido durante la consulta, el lenguaje es prácticamente ininteligible en el contexto de una conversación, pero si tiene que nombrar objetos, o nombres propios de la familia, se detiene y es más claro.

A partir de la evaluación en esta consulta, consideramos necesario volver a convocarlos para continuar con las evaluaciones y desplegar lo que aparece de la historia en el relato de la mamá. De esta manera ofrecemos un espacio particular y recortado para la entrevista con ella, y otro tiempo para el trabajo con el hijo. Pueden tomar esta oferta.

En la siguiente entrevista a solas, la madre refiere que su hijo se ha quedado enganchado con uno de los juegos propuestos en el dispositivo. El juego consiste en presentar objetos ocultos a la mirada. El niño tiene que adivinar de qué se trata a través del tacto y la manipulación. "Cuando nació la hermana más chica, él quería cambiarle los pañales, la cambia para ir al jardín. Con el otro hermano no se llevan bien, lo molesta y no reacciona, para que lo haga tiene que sacarle la paciencia”, continúa.

Sobre la historia temprana del niño, responde: "De bebé no lloraba, dormía mucho, lo despertaba yo mojándole la cara para darle la teta". "Cuando empezó a comer, yo lo dejaba que se ensuciara. En el corralito se trepa- 